\title{
Monte Carlo studies of the intrinsic time-domain response of nanoscale three-branch junctions
}

\author{
I. Iñiguez-de-la-Torre, ${ }^{1, a)}$ H. Rodilla, ${ }^{2}$ J. Mateos, ${ }^{1}$ T. González, ${ }^{1}$ H. Irie, ${ }^{3, b)}$ \\ and Roman Sobolewski ${ }^{1, \mathrm{c}}$ \\ ${ }^{1}$ Departamento de Física Aplicada, Universidad de Salamanca, Plaza de la Merced $s / n$, \\ E-37008 Salamanca, Spain \\ ${ }^{2}$ Microwave Electronics Laboratory, Department of Microtechnology and Nanoscience, Chalmers University \\ of Technology, SE-41296 Gothenburg, Sweden \\ ${ }^{3}$ Department of Electrical and Computer Engineering and Laboratory for Laser Energetics, University of \\ Rochester, Rochester, New York 14627-0231, USA
}

(Received 17 January 2012; accepted 7 March 2012; published online 27 April 2012)

\begin{abstract}
We present a Monte Carlo time-domain study of nanostructured ballistic three-branch junctions (TBJs) excited by both step-function and Gaussian picosecond transients. Our TBJs were based on InGaAs 2-dimensional electron gas heterostructures and their geometry followed exactly the earlier experimental studies. Time-resolved, picosecond transients of both the central branch potential and the between-the-arms current demonstrate that the bandwidth of the intrinsic TBJ response reaches the $\mathrm{THz}$ frequency range, being mainly limited by the large-signal, intervalley scattering, when the carrier transport regime changes from ballistic to diffusive. (C) 2012 American Institute of Physics. [http://dx.doi.org/10.1063/1.4704371]
\end{abstract}

\section{INTRODUCTION}

Electron devices that can operate in the terahertz $(\mathrm{THz})$ frequency electromagnetic spectrum (approx. 0.3 to $30 \mathrm{THz}$ ) have recently gained significant importance because of a wide variety of emerging applications. ${ }^{1}$ Among the candidates for $\mathrm{THz}$ electronics, nanostructures based on III-V high-mobility materials and utilizing ballistic electron transport have been regarded as one of the most promising because of their planar geometry with ultra-low internal capacitance and operation at room temperatures. ${ }^{2}$ One class of such room-temperature ballistic devices is nanoscale three-branch junctions (TBJs) based on InGaAs twodimensional electron gas (2DEG) heterostructures. ${ }^{3-7}$ The TBJ (with a T or Y shape) exhibits a well-known non-linear, bell-shaped response of the central branch's potential, when a push-pull voltage signal is applied to its arms. In the lowbias regime (typically for a voltage between the arms below approx. $\pm 0.5 \mathrm{~V})$, the response is parabolic because of the presence of ballistic or quasi-ballistic transport; whereas at high bias, the output has a linear dependence, attributed to the formation of a low-velocity domain when the applied voltage exceeds the $\Gamma-\mathrm{L}$ intervalley energy threshold. ${ }^{8,9}$ In large, micrometer-scale TBJs, only the second, diffusive regime has been observed. ${ }^{8}$

Most recently, some of us have experimentally demonstrated a sub-THz performance of a two-TBJ rectifier when

\footnotetext{
a) Author to whom correspondence should be addressed. Electronic mail: indy@usal.es.

b) Present address: NTT Basic Research Laboratories, Atsugi-shi, Kanagawa 243-0198, Japan.

c) On the sabbatical leave from the Departments of Electrical and Computer Engineering and Physics and Astronomy, Materials Science Program, and Laboratory for Laser Energetics, University of Rochester, Rochester, New York 14627-0231, USA. Secondary appointment: Institute of Electron Technology, PL-02668 Warszawa, Poland.
}

excited by single-picosecond electrical pulses. ${ }^{10}$ The measurements were performed in an "experiment-on-chip" configuration, with the TBJs integrated into a coplanar transmission line and the $\sim 1.8$-ps-wide excitation transient generated in-situ by a photoconductive switch. The output was detected in time-domain using an electro-optic sampling technique. ${ }^{11}$ Unfortunately, the transmission line configuration limited our dynamical studies only to measurements performed on the unbiased TBJs. In addition, only a small fraction of the excitation signal was actually coupled into the rectifier because of a severe impedance mismatch at the rectifier-transmission line interface. Therefore, the studies were done exclusively in the small-signal regime with only limited ability to directly observe the TBJ nonlinear response at $\mathrm{THz}$ frequencies.

Here, our aim is to complement time-domain experimental studies of the TBJ by means of Monte Carlo (MC) simulations of its transient response. Our approach uses an ensemble MC simulator coupled with a 2D Poisson solver, presented in detail in Ref. 12. First, in Sec. II, we replicate the experimental conditions of Ref. 10, thus validating our model. Next, in Sec. III, we study the large-signal regime of the TBJs subjected to both step and pulse voltages of different amplitudes applied to their terminals. To complete our studies we simulate a higher III-V mobility material, InAs, for the TBJ.

\section{VALIDATION OF THE MODEL}

Figure 1 shows a MC-simulated transfer $V_{\mathrm{C}}$ vs. $V_{1}$ characteristics obtained by calculating the floating potential at the central terminal $V_{\mathrm{C}}$ when biasing the TBJ in a push-fix fashion, i.e., sweeping the potential at the right terminal, $V_{1}$, with $V_{2}=0$. We note that we achieved very good agreement between our MC simulations (open dots) and the experiment 


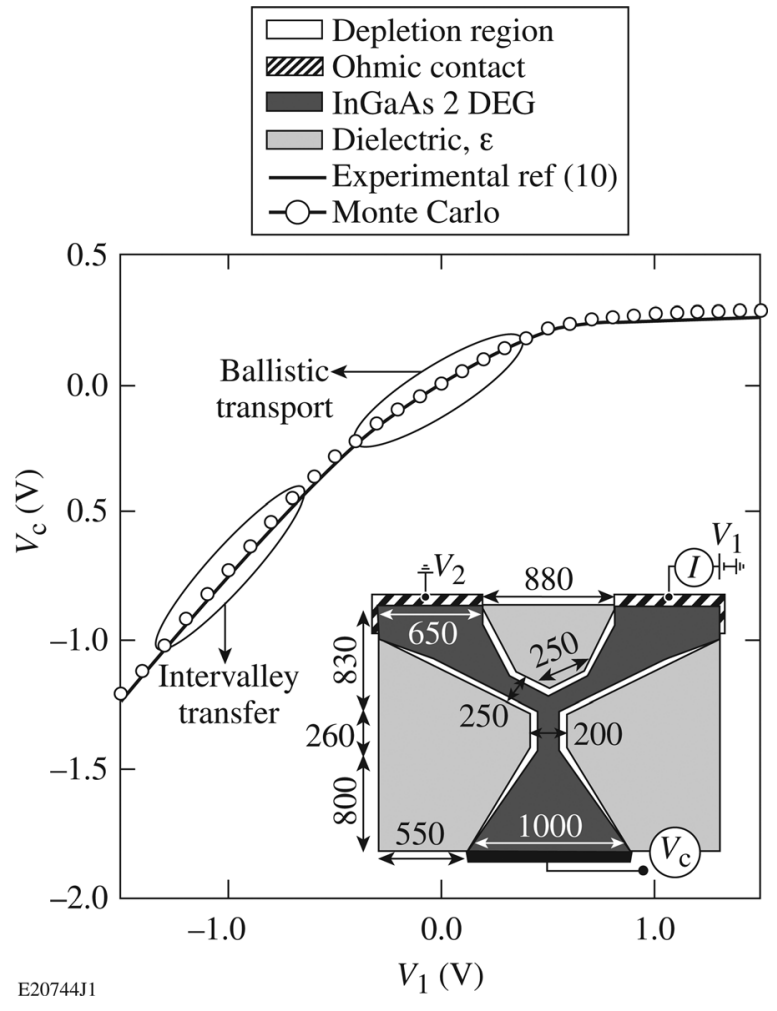

FIG. 1. MC-modeled TBJ transfer characteristics (open dots) of the central branch voltage $V_{\mathrm{C}}$ versus the right-hand branch voltage $V_{1}$ (push-fix fashion) with the ballistic and intervalley transfer regimes marked. Experimental data (solid line) are from Ref. 10. The inset shows the geometry and the biasing scheme of the TBJ used in the MC simulations, following the real TBJ of Ref. 10. The different device regions are labeled in the legend and all dimensions are in nanometers.

(solid line; see also Fig. 1 in Ref. 10). The well-known, nonlinear, rectifying behavior is caused by the quasiballistic electron transport at the low-bias regime, and the linear dependence observed in the high-bias region is due to inter-valley transfer $\left(V_{1}>0.5 \mathrm{~V} \approx \Delta E_{\mathrm{L} \Gamma} / q=0.45 \mathrm{~V}\right.$, the voltage difference between the bottoms of the $\mathrm{L}$ and $\Gamma$ valleys for InGaAs with $q$ the electron charge). The intervalley scattering of carriers is also responsible for the saturation regime in the TBJ's current-voltage $(I-V)$ characteristics (not shown). The inset in Fig. 1 presents a detailed geometry of the studied device, following the experimental $\mathrm{In}_{0.7} \mathrm{Ga}_{0.3}$ As-based TBJ tested in Ref. 10. The dimension of the active region is $\sim 200 \mathrm{~nm}$, and the fitting parameters used in the simulation are: surface charge density $\sigma / q=0.55 \times 10^{12} \mathrm{~cm}^{-2}$ and sheet-electron density in the active 2DEG layer $n_{\mathrm{s}}=6 \times 10^{11} \mathrm{~cm}^{-2}$. The angle between the left and right branches is around $60^{\circ}$.

\section{TIME-DOMAIN RESPONSE}

\section{A. Step function transients}

Our systematical time-domain MC analysis started with a simple step-like excitation in a push-fix biasing regime. Two sequences of stepped $V_{1}(t)$ voltage transients were applied [see Fig. 2 caption for the actual $V_{1}(t)$ waveforms], forcing the TBJ to undergo the transition between ballistic and intervalley-dominated regimes, indicated in Fig. 1. The

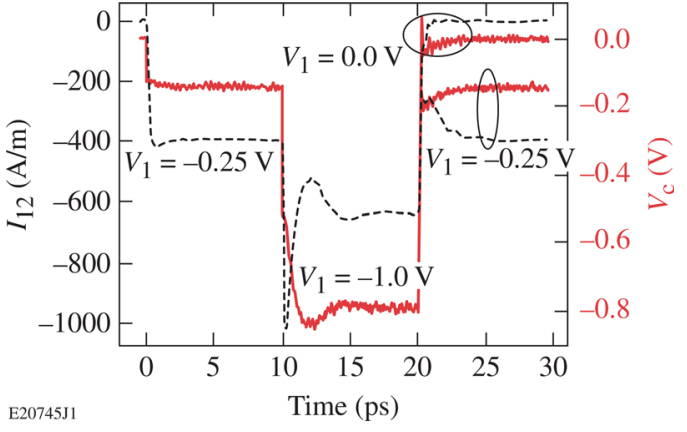

FIG. 2. MC-modeled time-resolved evolutions of $I_{12}(t)$ (black dashed line and left axis) and $V_{\mathrm{C}}(t)$ (red line and right axis) transients in response to two sequences of $V_{1}(t)$ voltage steps: $V_{1}(t)=[-0.25 \Theta(t)-0.75 \Theta(t-10 \mathrm{ps})$ $+0.75 \Theta(t-20 \mathrm{ps})] \mathrm{V}$ and $V_{1}(t)=[-0.25 \Theta(t)-0.75 \Theta(t-10 \mathrm{ps})$ $+1.00 \Theta(t-20 \mathrm{ps})] \mathrm{V}$, with $\Theta$ the Heaviside step function.

resulting MC-modeled, time-dependent values of the $I_{12}(t)$ current flowing between the arms and the $V_{\mathrm{C}}(t)$ stem voltage, plotted as a function of time, are shown in Fig. 2 (Ref. 13). First, we note that the time evolutions of the $I_{12}$ and $V_{\mathrm{C}}$ responses are different, since different processes have an influence on them. Although $I_{12}(t)$ comes mainly from the carrier transport (i.e., velocity) between the side branches, it takes an additional redistribution of the carrier population to reach the stationary value for $V_{\mathrm{C}}(t)$. Significant differences appear depending on the initial bias point and the amplitude of the excitation step voltage. First, for $V_{1}$ transition from 0 to $-0.25 \mathrm{~V}$, the responses of both $I_{12}$ and $V_{\mathrm{C}}$ are very fast, since we remain in the ballistic region (below the $\Delta E_{\Gamma \mathrm{L}} / q$ value) and electrons ballistically readapt to the driving voltage, reaching almost instantaneously the steady-state values within our 10-ps step window. When $V_{1}$ changes from -0.25 to $-1.0 \mathrm{~V}$ within the next 10 -ps window, the intervalley scattering becomes dominant and a clear nonequilibrium time evolution of both transients is observed. The $I_{12}(t)$ signal actually shows a pronounced peak followed by a slow dumped oscillation. The peak is due to the well-known in III-V materials the velocity overshoot effect, appearing at the initial stages of the high-voltage acceleration of electrons. ${ }^{14}$ After that, the electrons are subject to an intervalley scattering and undergo subsequent $\Gamma-\mathrm{L}$ and $\mathrm{L}-\Gamma$ transitions until the stationary conditions are reached. In III-V materials, the inter-valley scattering time is of the order of a few picoseconds, which is well reproduced in our simulation. The $V_{\mathrm{C}}(t)$ signal reflects the evolution of the nonequilibrium carrier population. Finally, we have subjected our TBJ to two different return routes: one from -1.0 to $-0.25 \mathrm{~V}$ and the other form -1.0 to $0 \mathrm{~V}$ (see Fig. 2 at $t>20 \mathrm{ps}$ ). For $V_{\mathrm{C}}(t)$, the return to the ballistic regime is in both cases rapid and very similar; the steady-state voltage is reached when excited carriers return to the $\Gamma$ valley. The $I_{12}(t)$ evolution is even more rapid when the final bias is set to $0 \mathrm{~V}$ since in the absence of driving force, the intense scattering after the voltage step is enough to reach the final zero-current value irrespectively of valley occupation. However, in the case of the $-0.25-\mathrm{V}$ bias, $I_{12}(t)$ reaches the steady-state value only after the energy relaxation of carriers through the $\mathrm{L}-\Gamma$ transitions and within the $\Gamma$ valley, which is relatively slow because of the low density of states (low effective mass) in the $\Gamma$ valley. 


\section{B. Gaussian picosecond transients}

In the second phase of our MC simulations, we have studied the pulsed excitation of our TBJ in the form of a Gaussian transient with a full width at half maximum (FWHM) equal to $0.25 \mathrm{ps}, 2 \mathrm{ps,}$ and $20 \mathrm{ps}$. Because the results for both the 0.25 - and 2-ps-wide excitations were qualitatively very similar, we focus our discussion here on the 2-ps pulses, experimentally much more realistic. For comparison, we have also calculated the static/steady-state responses using the transfer function shown in Fig. 1.

As expected from our step-function simulations, for pulse amplitudes within the low-bias ballistic regime (results not shown here), the TBJ $I_{12}(t)$ and $V_{\mathrm{C}}(t)$ responses replicated the static curves for 2-ps-wide Gaussians, while in the case the 0.25-ps excitation, an $\sim 1$-ps broadening was observed and the amplitude of the $I_{12}(t)$ signal was $\sim 70 \%$ of the steadystate value. In particular, we have modeled the conditions of Ref. 10 and applied a 60-mV-high and 1.8-ps-wide input pulse in our MC-simulator. The shape of the output transient was unchanged, confirming that the experimental shape of the TBJ response measured in Ref. 10 was dominated by capacitive elements of the TBJ incorporated into the transmission line. In fact, a simple circuit simulation test, in which we convoluted our "ideal" MC response with an external capacitive network of a TBJ rectifier, derived by Bednarz et al., ${ }^{7}$ resulted in a few-ps-long, single-oscillation transient that very closely resembled the experimental signal presented in Ref. 10.

Under the large-signal condition, we used $1-$ and $0.75-\mathrm{V}$ amplitude Gaussian inputs to transiently drive the TBJ from the ballistic regime (starting from $V_{1}$ values of 0 and $-0.25 \mathrm{~V}$, respectively) into the diffusive one and back [Fig. 3(a)] and a 1-V Gaussian in opposite direction [starting from $-1 \mathrm{~V}$, Fig. 3(b)]. We note that in Fig. 3(a), despite the large-signal switching regime, we observe no pronounced effects associated with the $\Gamma-\mathrm{L}$ transition on the $V_{\mathrm{C}}(t)$ transients that almost ideally follow the shape of the static pulse, being only slightly delayed, and stretched at the bottom of the trailing edge. The $I_{12}(t)$ current pulse, in turn, exhibits an ideal rising edge, but its falling edge is distorted. Exactly as explained in the case of the step-function simulation (Fig. 2), the $I_{12}(t)$ falling-edge shape depends on whether the excitation end state is the $0 \mathrm{~V}$ or $-0.25 \mathrm{~V}$ condition, having an overshoot and prolonged relaxation tail in the latter case. We also note that the $I_{12}(t)$ waveform significantly differs from the corresponding steady-state solution, which exhibits a flat top caused by to the current saturation of the TBJ $I-V$ characteristics in the diffusive regime. An $I_{12}(t)$ dynamical transient exactly following the static flattop solution could be reproduced when the input excitation was, e.g., 20-ps wide.

When the initial bias of our TBJ was $V_{1}(0)=-1 \mathrm{~V}$ [Fig. 3(b)], electrons were already present in the $L$ valley and the impact of the inter-valley scattering dynamics was clearly visible on the falling (corresponding to the ballistic-to-diffusive transition) edges of the $I_{12}(t)$ and $V_{\mathrm{C}}(t)$ transients. As in the case of stepped transients, the most-pronounced oscillations were observed in the $I_{12}(t)$ waveform, and interestingly, their period was only material related, being exactly the same in the case of 0.25 - and 2-ps-wide excitations. The latter strongly

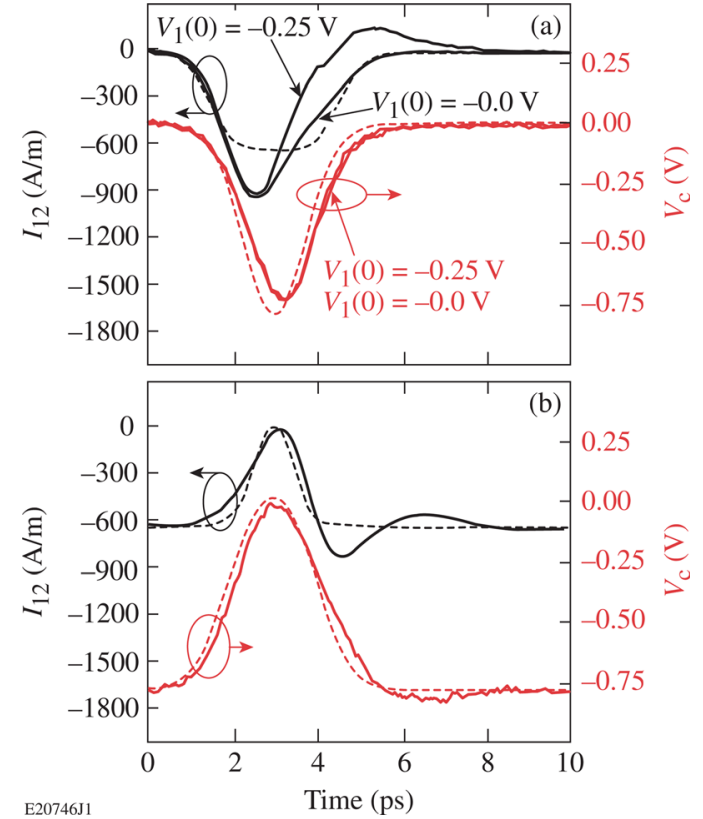

FIG. 3. MC-modeled time-resolved evolutions of $I_{12}(t)$ (black lines and left axis) and $V_{\mathrm{C}}(t)$ (red lines and right axis) transients in response to 2-ps-wide Gaussian pulses. The dashed-line traces correspond to the static/steady-state solutions. (a) Transitions from the ballistic regime with $V_{1}(0)$ values of 0 and $-0.25 \mathrm{~V}$ into the diffusive one $\left(V_{1}=-1 \mathrm{~V}\right)$ and back. In order to enable a direct comparison, the pulses corresponding to $V_{1}(0)=-0.25 \mathrm{~V}$ are amplitude scaled $\left(I_{12}\right.$ by the factor of 2.413 and $V_{\mathrm{C}}$ by 1.2) and shifted to 0 by $-391 \mathrm{~A} / \mathrm{m}$ and $-0.151 \mathrm{~V}$. (b) Transition from the diffusive, $V_{1}(0)=-1 \mathrm{~V}$, regime into the ballistic one $\left(V_{1}=0 \mathrm{~V}\right)$ and back.

indicates that the best way to improve the TBJ ultrahighfrequency performance is to fabricate it using a material with high mobility and large $\Gamma-\mathrm{L}$ energy separation, such as InAs (Refs. 15 and 16) rather than, e.g., shrinking its dimensions to enhance ballisticity.

To corroborate the expected improvement in the ultrahigh-frequency performance of TBJs fabricated in the higher mobility materials as, e.g., InAs, we present in Fig. 4 the $I_{12}(t)$ and $V_{\mathrm{C}}(t)$ transients simulated under the same conditions as in Fig. 3(b) but for an InAs-based TBJ. The microscopic material parameters used in these MC modeling are available in Ref. 17. It is clearly observed that the responses of both waveforms are strongly dumped, and their respected stationary values are reached much sooner (within $\sim 2 \mathrm{ps}$ ) than for the case of the InGaAs-based TBJ [Fig. 3(b)]. The latter is because of the higher velocity and lower effective

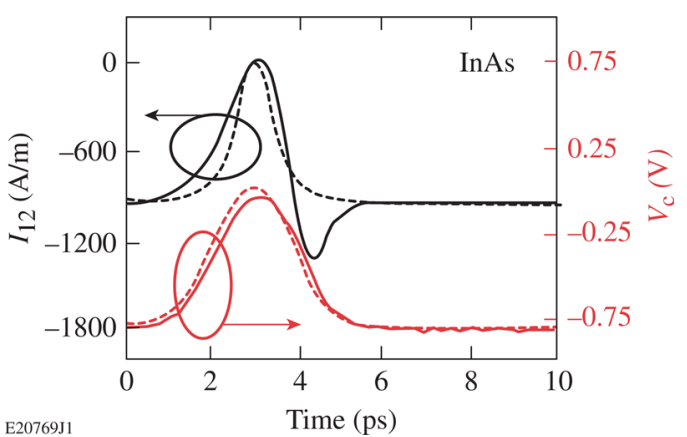

FIG. 4. MC-modeled time-resolved transients, the same as signals in Fig. 3(b), but simulated for an InAs-based TBJ. 
mass (0.023) of electrons in the InAs $\Gamma$ valley, as well as the fact that in the case of the InAs TBJ, the applied $I-V$ Gaussian pulse is not enough to initiate the carrier transfer into the upper valley, since $\Delta E_{\mathrm{L} \Gamma} / q=1.1 \mathrm{~V}$.

\section{CONCLUSIONS}

In summary, our Monte Carlo time-resolved simulations of nanostructured InGaAs TBJs excited by both the stepfunction and Gaussian picosecond transients revealed that the bandwidth of the intrinsic TBJ response extended into the $\mathrm{THz}$ range, being mainly limited by a few-ps-in-duration, $\Gamma-\mathrm{L}$ intervalley scattering. Pronounced oscillations were observed in the $I_{12}(t)$ transport current response during the TBJ switching from the ballistic into the diffusive regime and were interpreted as the velocity overshoot effect present in III-V materials when electrons are accelerated and transition from $\Gamma$ to $\mathrm{L}$ valley takes place. The central stem $V_{\mathrm{C}}(t)$ response was almost undistorted, confirming that the TBJ could be implemented as, e.g., a THz-rate "AND" gate. For low-signal excitations, within the ballistic regime, the timeresolved TBJ response practically followed the excitation signal, confirming the earlier, experimental notion that in such case the main distortion comes from external capacitive network surrounding the TBJ placed in the transmission line. For excitation pulses wider than $20 \mathrm{ps}$, the TBJ response signal, even in the large-signal regime, simply followed the steady-state solution. Based on our studies, we can speculate that TBJs fabricated using materials characterized by ultrahigh mobilities and the absence of the intervalley scattering, e.g., graphene, should exhibit intrinsically a frequency response well in the $\mathrm{THz}$ regime.

\section{ACKNOWLEDGMENTS}

The authors thank Martin Margala for long-term collaboration and extended discussions. Work in Salamanca was partially supported by the European Commission through the
ROOTHz Project ICT-2009-243845, by the Dirección General de Investigación (MICINN) through Project TEC201015413, by the Consejería de Educación, Junta de Castilla y León through Project GR270. Work in Rochester was supported in part by the NSF Nanotechnology Interdisciplinary Research Team grant No. ECCS-0609140. R.S. thanks the Spanish Ministry of Education and the University of Salamanca for their financial support (through grant SAB20100003) and hospitality during his one-semester-long (fall 2011) research visit in Salamanca, Spain.

\footnotetext{
${ }^{1}$ M. Tonouchi, Nat. Photonics 1, 97 (2007).

${ }^{2}$ A. M. Song, Encyclopedia Nanosci. Nanotechnol. 9, 371 (2004) and references therein.

${ }^{3}$ K. Hieke and M. Ulfward, Phy. Rev. B, 62, 16727 (2000).

${ }^{4}$ H. Q. Xu, Appl. Phys. Lett. 78, 2064 (2001).

${ }^{5}$ L. Worschech, A. Schliemann, S. Reitzenstein, P. Hartmann, and A. Forchel, Microel. Eng. 63, 217 (2002).

${ }^{6}$ I. Shorubalko, H. Q. Xu, I. Maximov, P. Omling, L. Samuelson, and W. Seifert, Appl. Phys. Lett. 79, 1384 (2001).

${ }^{7}$ L. Bednarz, Rashmi, B. Hackens, G. Farhi, V. Bayot, and I. Huynen, IEEE Trans. Nanotechnol. 4, 576 (2005).

${ }^{8}$ J. Mateos, B. G. Vasallo, D. Prado, T. Gonzales, E. Pichonat, J. S. Galloo, S. Bollaert, Y. Roelens, and A. Cappy, IEEE Electron Device Lett. 25, 235 (2004).

${ }^{9}$ H. Irie, Q. Diduck, M. Margala, R. Sobolewski, and M. J. Feldman, Appl. Phys. Lett. 93, 053502 (2008).

${ }^{10} \mathrm{H}$. Irie and R. Sobolewski, J. Appl. Phys. 107, 084315 (2010).

${ }^{11}$ J. A. Valdmanis, G. A. Mourou, and C. W. Gabel, Appl. Phys. Lett. 41, 211 (1982).

${ }^{12}$ J. Mateos, B. G. Vasallo, D. Pardo, T. González, J. S. Galloo, S. Bollaert, Y. Roelens, and A. Cappy, IEEE Trans. Electron Dev. 50, 1897 (2003).

${ }^{13}$ The symmetry of the TBJ $I-V$ characteristics assures that positiveamplitude $V_{1}(t)$ excitations would result in physically the same behavior as the ones presented in Fig. 2.

${ }^{14}$ J. Son, W. Sha, J. Kim, T. B. Norris, J. F. Whitaker, and G. A. Mourou, Appl. Phys. Lett. 83, 923 (1993).

${ }^{15}$ D. B. Suyatin, J. Sun, A. Fuhrer, D. Wallin, L. E. Fröberg, L. E. Karlsson, I. Maximov, L. R. Wallenberg, L. Samuelson, and H. Q. Xu, Nano. Lett. 8 1100 (2008).

${ }^{16}$ G. M. Jones, C. H. Yanga, M. J. Yang, and Y. B. Lyanda-Geller, Appl. Phys. Lett. 86, 073117 (2005).

${ }^{17}$ H. Rodilla, T. González, D. Pardo, and J. Mateos, J. Appl. Phys. 105, 113705 (2009).
} 